pared with the brain receptor. Further medicinal chemistry efforts could no doubt yield highly selective ligands. It is possible that such compounds might represent novel anti-inflammatory or immunosuppressant agents. The discovery by Munro et al. highlights yet again the ability of molecular biology to discover new receptors at a rate much faster than pharmacologists can handle.

Leslie L. Iversen is at Merck, Sharp \& Dohme Research Laboratories Neuroscience Research Centre, Terlings Park, Eastwick Road, Harlow, Essex CM2O 2QR, UK

\title{
Twice is not enough
}

\section{Kevin Hurley}

THE heavens are anything but immutable at high energies. Sources of X-rays and gamma-rays come and go on all timescales. The most fleeting high-energy phenomena are the X-ray bursters, the soft gamma repeaters and the gamma-ray bursters. The X-ray bursters are fairly well understood in terms of thermonuclear explosions and accretion onto neutron stars, the dense remnants of massive but otherwise normal stars which have finished their lives by exploding as supernovae. In contrast, little is known about the soft gamma repeaters or the gamma-ray bursters, largely because until now no one has been able to find a completely convincing quiescent counterpart to either in any part of the electromagnetic spectrum. Now Kulkarni and Frail, on page 33 of this issue, have announced the discovery of a supernova remnant whose location is consistent with that of a soft gamma repeater.

Observationally, the three classes of high-energy transients are easy to distinguish. The three dozen or so known X-ray bursters have soft spectra, typical of blackbodies with temperatures of about $1 \mathrm{keV}$ coming from a surface area of a few square kilometres, and the sources are observed to burst repeatedly over timescales of years. Only three soft gamma repeaters are known, and their energy spectra may be characterized by that of a hot gas (thermal bremsstrahlung) at a temperature of $40 \mathrm{keV}$. One, SGR1806-20, has been observed to burst well over 100 times.

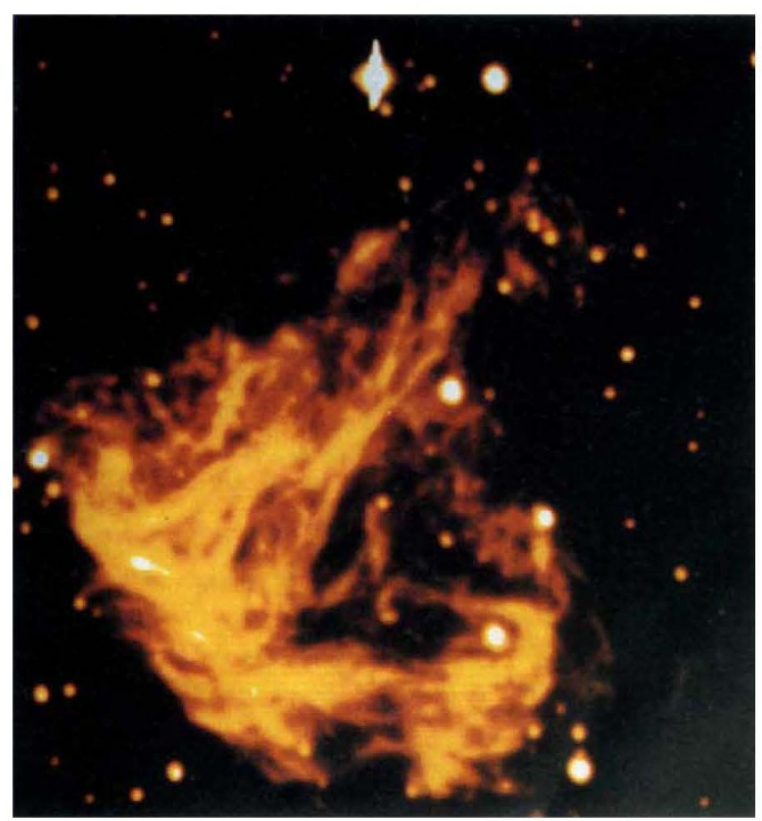

The N49 supernova remnant in the Large Magellanic Cloud This remnant probably contains a neutron star which generated the very intense 1979 March 5 burst, and 16 subsequent weaker events. The radio supernova remnant reported by Kulkarni and Frail lies in a highly obscured region, towards the Galactic Centre, and cannot be detected at optical wavelengths.
Another, the source of the bright anomalous 1979 March 5 event, has burst 17 times. The third, B1900+14, burst three times in three days in 1979 and three times in two months in 1992. Finally, the gamma-ray bursters have hard energy spectra, which have been measured to over $100 \mathrm{MeV}$. More than a thousand have now been observed, and there is no compelling evidence that any single source has burst more than once.

There has been no consensus on the origin of either the soft gamma repeaters or the gamma-ray bursters. Indeed, various theories have placed them at distances of a few hundred parsecs or throughout the depths of the Universe. A tantalizing clue in this puzzle was that the location of the 1979 March 5 source is consistent with a supernova remnant, N49. However, because it is in the Large Magellanic Cloud at a distance of $55 \mathrm{kpc}$,

ron stars in supernova remnants.

Kulkarni and Frail's announcement that the position of the galactic supernova remnant $\mathrm{G} 10.0-0.3$ is consistent with that of SGR1806-20 supports this view. So we now have two identifications of soft gamma repeaters that point to a neutron star origin in a young (less than 10,000 years old) supernova remnant, and many, many ideas about what may cause them to burst, from accretion to starquakes. But there are probably several hundred such remnants in the Galaxy, and only three soft gamma repeaters, even though the soft gamma repeaters seem to be bright enough to be detected by current instruments whatever their distance, as long as it is galactic. Kulkarni and Frail speculate that only neutron stars with certain properties - magnetic field strength and rotation rate in a certain range, for exampleevolve to become soft gamma repeaters. And they may not remain in this phase for very long, so it could well be that three or so is indeed the total number that are currently active.

What of the third soft gamma repeater, B1900+14? Its location is nowhere near as precisely known as the other two, and there are no known supernova remnants precisely in its error box. But if guilt by association can be accepted as evidence, then this too can plausibly be assumed to be related to a neutron star in a young, galactic supernova remnant. Proof may be forthcoming if current searches bear fruit. This may well lay to rest the cosmological theories of the origin of the soft gamma repeaters.

And the ultimate mystery, the gamma-ray bursters? The genealogy of high-energy transient sources is too poorly known to conclude that soft gamma repeaters and gamma-ray bursters are related, although there have been theories to this effect. For one thing, even if every neutron star in the Galaxy were a gamma-ray burster, there would still not be enough to have each one burst only once. On some timescale, perhaps hundreds of thousands of years or more, they would have to repeat. For another, the light curves and energy spectra of the two classes are vastly diffe-

an exceptionally large luminosity, $5 \times 10^{44}$ erg s $\mathrm{s}^{-1}$, is implied. Thus theorists have been led to sources either more mundane (nearby neutron stars) or more exotic (multiple, gravitationally lensed images of a single explosion at cosmological distances). Still, the apparent directions of the two other soft gamma repeaters - near the Galactic Centre for SGR1806-20, and close to the galactic plane for B1900+14 - hinted at a unified explanation for all three sources as neutrent. Although such objections can always be overcome by evoking source evolution, there is still no hard evidence to link them. So for the time being, the identification of two out of three soft gamma repeaters appears to solve only the second of three mysteries surrounding the origin of highenergy, short-timescale transients.

Kevin Hurley is at the Space Sciences Laboratory, University of California, Berkeley, California 94720, USA. 